\title{
Color Influence and Genetic Algorithm Optimization in Interior Lighting Building
}

\author{
Merimé Souffo Tagueu*, Benoît Ndzana \\ Laboratoire de Génie Electrique, M’catronique et Traitement du Signal, National Advanced School of Engineering, University of Yaoundé I, \\ Yaoundé, Cameroon
}

Email address:

souffotagueu@yahoo.fr (M. S. Tagueu)

${ }^{*}$ Corresponding author

\section{To cite this article:}

Merimé Souffo Tagueu, Benoît Ndzana. Color Influence and Genetic Algorithm Optimization in Interior Lighting Building. American Journal of Electrical Power and Energy Systems. Vol. 8, No. 6, 2019, pp. 165-175. doi: 10.11648/j.epes.20190806.14.

Received: October 28, 2019; Accepted: November 20, 2019; Published:Published: December 30, 2019

\begin{abstract}
The energy consumed by the lighting of the buildings represents a not negligible part of the total energy. The use of low-energy luminaires such as LEDs has significantly reduced this consumption, in addition to the reduction of greenhouse gases and the extended life of the lamps. To satisfy the basic principles of optimal lighting system design (i.e., maximizing uniformity and reducing the level of illumination by staying within the required normative range), many researches using optimization algorithms have been conducted with interesting results. This article proposes a multi-objective optimization model integrating the influence of the colors (in particular primary colors), of the different compartments of a room on the level of total illumination of the piece. The reduction of energy consumption is demonstrated by considering a specific model of illumination in which we introduced the reflection factor related to the colors of the surrounding environment. The subsequent use of genetic algorithms (NSGA III) makes it possible to find the optimal coefficient of variation of the LEDs or any other variable luminaires to have the desired energy value while keeping the same comfort for the users. The proposed model is implemented for the case of an office room. The results show an energy savings of up to $39 \%$ with red color. Of particular, results are obtained while maintaining regular illumination and changing the color of the pieces.
\end{abstract}

Keywords: Illumination, Multi-objective Optimization, Color, NSGA III, Energy Savings

\section{Introduction}

By 2050, our planet will have about 10 billion human beings, some countries including India, Nigeria, the United States, Indonesia, Brazil, Cameroon, just to name few, will see their population grow in a very important way. According to the International Energy Agency, the world's non-renewable energy reserves ( i.e. fossil and uranium) would give us an autonomy of 80 years of production at the current rate [1]. Such statistics invites us to revisit our consumption patterns or, to rethink how buildings, schools, and homes are built. Clearly, novel mind set on our daily habits in consuming energy should be defined. Present statistics reveals that $40 \%$ of the total energy produced in Europe is consumed solely by building[2, 3]. While in Africa, this percentage is larger, about 56\% [4].
Artificial lighting contributes for a significant portion (around $20-45 \%$ ) in overall energy consumption in office buildings[?].

Several studies have been conducted to reduce the energy consumption of buildings in the medium term (2020) and long term (2050)[6]. Some of them were focused on optimizing the type of construction, showing the energy savings related to the type of glazing [7, 8], the shape of the building [9-12], its geographical position[13], the type of building material [14], just to name few. Where others were related to the type of equipment by addressing consumer pockets such as cooling [15], ventilation [16-18], heating [15, 19, 20], lighting [21-24], and many others.

Regarding lighting, literature proposes a larger number of relevant works which suggest to reduce the amount of energy consumed in buildings [23, 25-28]. For instance, 
LED technology is used as a replacement for fluorescent luminaires, this has resulted in a considerable saving in power consumption, plus a longer luminaire life cycle[25]. Also, this LED technology provides the possibility of adjusting its level of illumination according to the needs of users.In the same line of minimizing the consumption, several methods with defined reduction criteria have been tested satisfactorily on LED luminaires. One can cite the particle swarms which were used to reduce the consumption of luminaires [23]. The optimal position of some luminaires was obtained using neural networks[26]. Also genetic algorithms were used to adjust the lighting level of fixtures with savings of up to $22 \%$ in energy consumed [27]. However, to the best of our knowledge, there is no work in the literature taking into account in addition to the variation of the levels of illumination, the impacts related to the surrounding colors (i.e. wall, ceiling and ground floor).

In this article, the impact of colors is studied to highlight aspects related to energy efficiency according to the type of color. This study considered optimization algorithms, in particular, the Non-Dominated Sorting Genetic Algorithm III (NSGA III), in other to show a relevant reduction in the percentage of dimming level of the luminaires.

The article is organized as follows: in section 2 , the color characterization is presented, followed by a mathematical reminder on the multi criterion optimization and the formulation of this optimization for the case of office lighting. Also, implementations of the NSGA III optimization method are provided. Section 3 presents a case study where the methods explained in the previous section are implemented to have the desired levels of illumination. The lighting optimization solution is shown in section 4 while conclusions and future works are drawn in section 5.

\section{Methodology}

\subsection{Model Formulation}

\subsubsection{Color Specificity}

By definition, color is the perception we have of the different wavelengths that constitute visible light. For an object, it depends on the nature of the light that illuminates, the nature of the illuminated object, and the characteristics of the eye and the brain. Most of the colors obtained are the result of mixing several primary colors, which can not be obtained by mixing other colors.

Primary colors are defined by two characteristics: (1) any primary color cannot be obtained by the combination of the two others, (2) Combining the three primary colors can produce a wider range of colors than using any other three colors. In the traditional color wheel (see Figure 1a), which is used for painting, the primary colors are red, yellow and blue. These colors were chosen apparently because of their vivid distinctness [29]. From the primary colors, secondary colors are obtained through the following combinations: red and yellow produces orange, then blue and yellow produces green while, mixing red and blue produces purple, as presented by the color wheel(see Figure $1 \mathrm{~b}$ ). The colors obtained after this mixture are called secondary colors.

The independence of the primary color to another color make our experiment to be more valuable. Indeed, the result would be more significant when considering colors which cannot be obtained through a mixture. The result given by the secondary or tertiary color will be within a known interval that the primary color associated with the neutral color (white or black) can give us.

\subsubsection{Optimisation Problem Formulation \\ Generalities}

The optimization problem is defined as the research of minimum or maximum of a giving function. This optimization need comes from the necessity of engineers to give the best solution to a problem to users according to some particular specifications.

Mathematically, an optimization problem is presented as follows :

$$
\left\{\begin{array}{c}
\text { minimise } J(\vec{x}) \\
\text { subject to } \vec{g}(\vec{x}) \leq 0 \\
\text { and } \vec{h}(\vec{x})=0
\end{array}\right.
$$

Where $\vec{x} \in R^{n}, \vec{g}(\vec{x}) \in R^{m}$, and $\vec{h}(\vec{x}) \in R^{p}$,

Here, vectors $\vec{g}(\vec{x})$ and $\vec{h}(\vec{x})$ respectively represent $\mathrm{m}$ inequality constraints and $\mathrm{p}$ equality constraints. These constraints bound a space to find optimal solutions.

In general, two types of constraints of inequality exist :

(1) The constraints of type $B_{\text {iinf }} \leq x_{i} \leq B_{\text {isup }}$ : the value of $\vec{x}$ that satisfies these constraints define the research space ;

(2) The constraints of type $C(\vec{x}) \leq 0$ or $C(\vec{x}) \geq 0$ : the value of $\vec{x}$ that satisfies these constraints define the realisable space values.

\section{Problem formulation}

There are two main characteristics in interior lighting namely illuminance and uniformity of the lighting. Both characteristics are normalized using EN 12464 standard [30] henceforth, turns all optimization efforts to lie within the specifications of the corresponding norm. It is worth to recall that our goal is to minimize energy consumption by reducing the dimming level of the luminaires in an interior building. Based on the superposition theory in physics, the illuminance at a specific point of the work plane level $\left(E_{i}\right)$, is equal to the contribution of each luminaire on that measurement point $\left(\omega_{i j}\right)$ and the corresponding dimming level of that luminaire $\left(\alpha_{j}\right)$ [27, 31-33].

$$
E_{i}=\sum_{j=1}^{N} \alpha_{j} \omega_{i j}
$$

where $\mathrm{N}$ is the number of luminaires and $\mathrm{i}$ depend on the number of measurements point $\mathrm{M}$

The mean illuminance, according to equation (2) is : 


$$
E_{m}=\frac{\sum_{i=1}^{M} E_{i}}{M}=\frac{\sum_{i=1}^{M} \sum_{j=1}^{N} \alpha_{j} \omega_{i j}}{M}
$$

To find the contribution of each luminaire, a simulation tool is used, but another method was to used a luxmeter at different choice points [27]. The luminaire needs to be already installed when the second method is used. The real value of illuminance can be found by introducing a specific factor $\rho_{k}$ due to color effect in different parts of a piece. This coefficient influences the final value of $\omega_{i j}$ as follows :

$$
\omega_{i j}=\rho_{k} \omega_{i j}^{\prime}
$$

where $\omega_{i j}^{\prime}$ is the value of measure illumination with a neutral color.

The factor $\rho_{k}$ is function of some specific boundary elements (ceiling, wall, floor) that constitute a room. Mathematically there exist a function such that :

$$
\rho_{k}=f\left(\rho_{c}, \rho_{w}, \rho_{f}\right)
$$

where $\rho_{c}, \rho_{w}, \rho_{f}$ are respectively the color effect factor of ceiling, wall, and floor.

By considering (4), equation (3) becomes :

$$
E_{m}=\frac{\sum_{i=1}^{M} \sum_{j=1}^{N} \alpha_{j} \rho_{k} \omega_{i j}^{\prime}}{M}
$$

The maximization of the uniformity of the illumination is obtained by minimizing the coefficient of the variation of the root mean square error $[27,34]$. This coefficient of variation $\left(\Delta E^{\prime}\right)$ is given by :

$$
\Delta E^{\prime}=\frac{\sqrt{\frac{\sum_{i=1}^{p} \sum_{j=1}^{q}\left(E_{i j}-E_{m}\right)^{2}}{p \times q}}}{E_{m}}
$$

where $\mathrm{p}$ and $\mathrm{q}$ are the measurement points along the two dimensions of the room.

Introducing equation (2) and (6) in (7),

$$
\Delta E^{\prime}=\frac{M}{\sqrt{p \times q}} \times \frac{\sqrt{\sum_{i=1}^{M}\left(\sum_{j=1}^{N} \alpha_{j} \rho_{k} \omega_{i j}^{\prime}-\frac{\sum_{i=1}^{M} \sum_{j=1}^{N} \alpha_{j} \rho_{k} \omega_{i j}^{\prime}}{M}\right)^{2}}}{\sum_{i=1}^{M} \sum_{j=1}^{N} \alpha_{j} \rho_{k} \omega_{i j}^{\prime}}
$$

The uniformity $g_{0}$ is defined as :

$$
g_{0}=\frac{E_{\text {min }}}{E_{m}}
$$

where $E_{\min }$ is the minimum value of illumination

\subsection{Optimization Algorithm}

Relevant literature in building optimization shares the common point on the fact that the genetic algorithms are particularly adapted in building design $[35,36]$ and fast than other heuristics [12]. They are easy to implement and do not easily get trapped in local minima. All these advantages oriented our choice on genetic algorithms throughout this paper. A multi-objective optimization algorithm namely Non Dominated Sorting Genetic Algorithm III (NSGA III) proposed by Deb et al. [37] is considered due to its performances on real-world problems. Recently NSGAIII has been frequently used for performance comparison of newly proposed evolutionary many-objective optimization algorithms [38, 39].

Genetic algorithms are all based on Darwin's principle of evolution. A population subjected to a hostile environment evolves over several generations following the law of natural selection. Clearly, best individuals which are adapted to an environment have the best chance of surviving and reproducing. Their legacy are then transmitted through their genes to the next generation. After several generations, the population obtained is more adapted to its environment than the initial population. Genes can also mutate and thus help to create more efficient individuals during evolution.
In practice, setting up a genetic algorithm first requires defining a representation of the variables, also called encoding. The attainment of a predetermined maximum number of generations or the one obtained after a certain number of generations with a degree of uniformity of the individuals of the population above a desired threshold is one of the stopping criteria that can be chosen for the algorithm. Figure 2 shows the flowchart of the proposed workflow methodology. Data is first collected, while lighting simulation is the second step. The optimization using NGSA III is then used to find the appropriate dimming level of the luminaires. The coefficient obtained is used in lighting simulation to verify if the required conditions are found. If no, optimization simulation is done again until the satisfactory condition found. If yes, we end the algorithm and move to analyze and conclusion.

\section{Implementation of the Methodology}

\subsection{Case Study: Office Lighting}

The optimization procedure is tested on an office room with dimensions of $5 \times 4.5 \times 2.8 \mathrm{~m}$. The color of the compound (wall, floor, and ceiling) is modified to appreciate the variation of illuminance. Primary colors (red, blue and yellow) and secondary colors (orange, purple and green) are chosen for different experimentation with chosen initial color as white or black. The other colors are not chosen because of their dependence on primary and secondary colors. The LED luminaire DN450B 1xDLM2000/840 of constructor PHILIPS is chosen for experimentation. Their specifications are shown 
in Table 1 while Figure 3 shows its image on the market. These luminaire are DALI controlled. The Digital Addressable Lighting Interface(DALI) is a standardized protocol (IEC 62386) for digital communication between lighting fixtures being addressed individually [40]. A simple 2-wire control line, which can also be pulled with the power line in a five- wire line under sheath, is sufficient for installation. All the LED luminaires used are dimmable and can be controlled by a central controlling computer. The dimming level of a luminaire is proportional to its power consumption. Figure 4 shows the implantation principle of the luminaire in an office room.

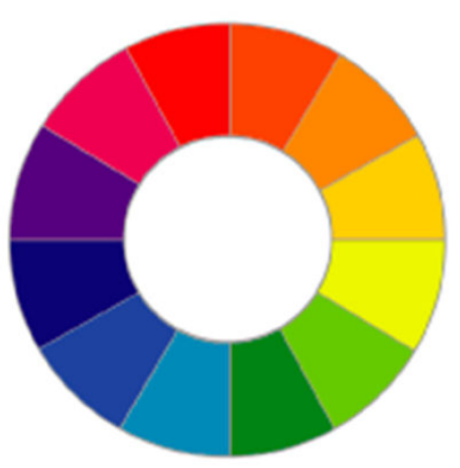

(a)

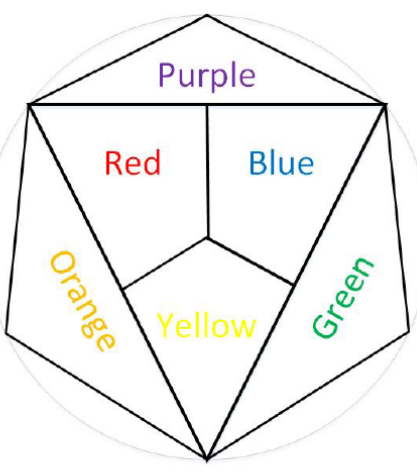

(b)

Figure 1. (a)The color wheel containing primary, secondary and tertiary colors. (b)The primary and secondary colors.

Table 1. Luminaire specifications.

\begin{tabular}{ll}
\hline Light Output Radio & 1.00 \\
Total luminaire power & $25 \mathrm{~W}$ \\
Total luminaire flux & $20001 \mathrm{~m}$ \\
Color temperature & $4000 \mathrm{~K}$ \\
Color Rendering Index & $>80$ \\
Average Lifetime & 50,000 hours \\
Technologie & LED \\
Dimmable & DALI \\
Manufacturer code & DN450B \\
\hline
\end{tabular}

Table 2. Illumination value according to color choice without optimization.

\begin{tabular}{lll}
\hline Color & $E_{m}$ & $g_{0}$ \\
\hline White or Black & 554 & 0.546 \\
Red & 818 & 0.783 \\
Blue & 595 & 0.703 \\
Yellow & 573 & 0.694 \\
Green & 605 & 0.708 \\
Purple & 610 & 0.711 \\
Orange & 620 & 0.721 \\
\hline
\end{tabular}

\subsection{Office Simulation}

DIALux is a simulation tool used by professionals for electric lighting design practice and energy research[41]. The illumination and uniformity values are fixed by the norm EN 12464 [30]. Henceforth, the recommended minimum values of illumination $E_{m}$ and uniformity $g_{0}$ are respectively $500 l x$ and 0.6. The Light loss factor is fixed at 0.8 and the height of the working plane around $0.800 \mathrm{~m}$.

Figure 5 shows the illumination isolines for primary colors including white and black color without specific optimization while Table 2 presents the different results in terms of illumination $E_{m}$ and uniformity $g_{0}$ without optimization. One can observe that illumination and uniformity increase when using primary and secondary colors. The maximum value of illumination is obtained with a red color. It is important after that to optimize these obtained illumination values to reduce energy consumption. Figure 6 shows the 3-D rendering of different illuminations.

\subsection{Optimization Procedure}

The mathematical formulation of the proposed multiobjective optimization, which is adapted to the arrangement of the above-mentioned office room is presented as : 


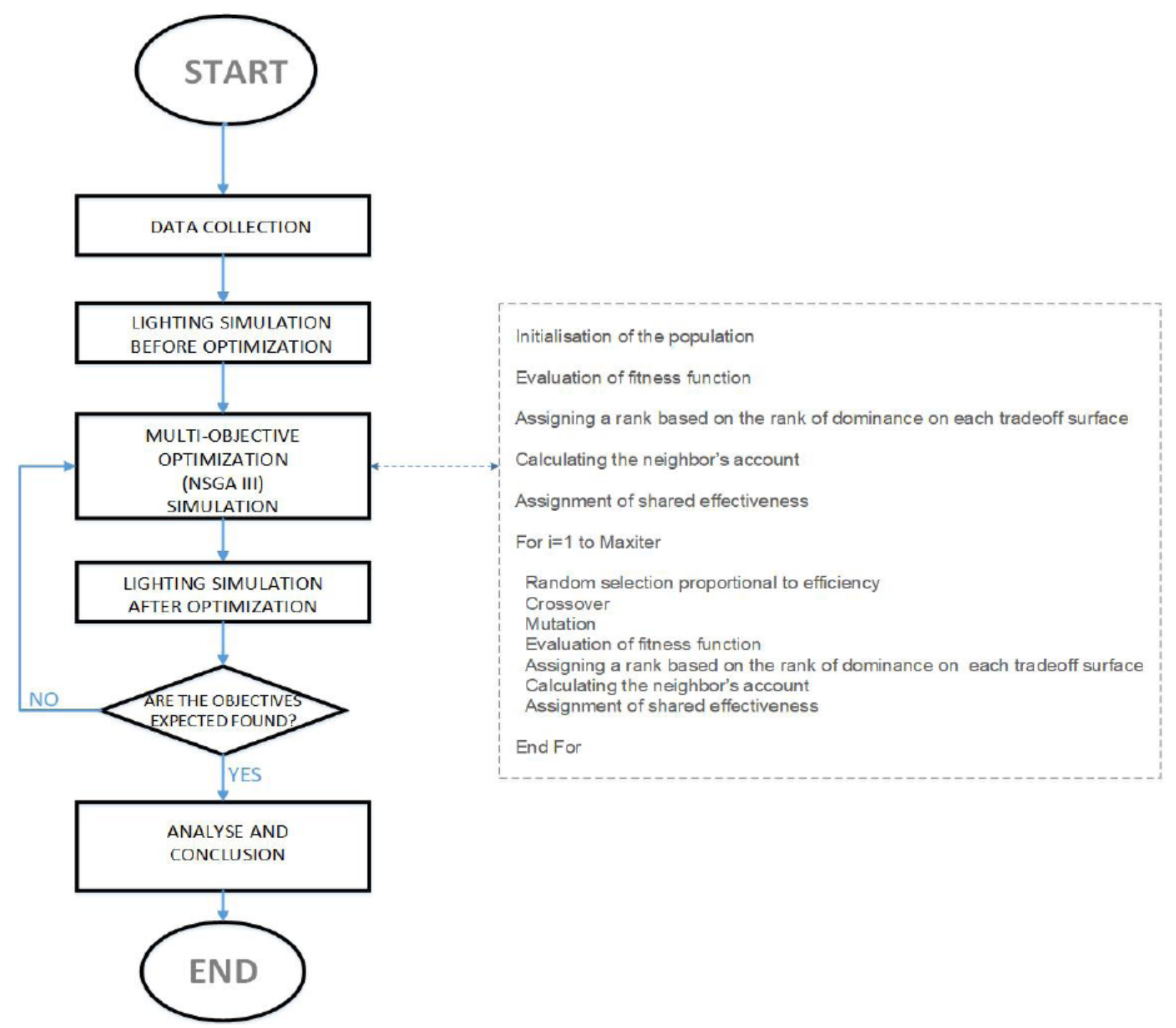

Figure 2. Flowchart for the simulation environment architecture.

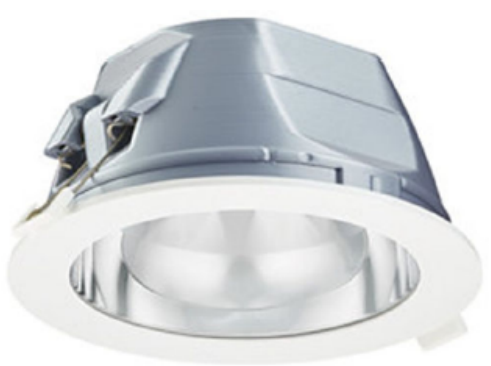

Figure 3. LED luminaire DN450B 1xDLM2000/840 of constructor Philips.

$$
\begin{cases}\text { minimise } & J_{1}(\alpha)=\sum_{k=1}^{9} \alpha_{k} \\ \text { minimise } & J_{2}(\alpha)=\frac{48}{\sqrt{8 \times 6}} \times \frac{\sqrt{\sum_{i=1}^{48}\left(\sum_{j=1}^{9} \alpha_{j} \rho_{k} \omega_{i j}^{\prime}-\frac{\sum_{i=1}^{48} \sum_{j=1}^{9} \alpha_{j} \rho_{k} \omega_{i j}^{\prime}}{48}\right)^{2}}}{\sum_{i=1}^{48} \sum_{j=1}^{9} \alpha_{j} \rho_{k} \omega_{i j}^{\prime}} \\ \text { minimise } & J_{3}(\alpha)=-\frac{48 \times E_{m i n}}{\sum_{i=1}^{48} \sum_{j=1}^{9} \alpha_{j} \rho_{k} \omega_{i j}^{\prime}}\end{cases}
$$




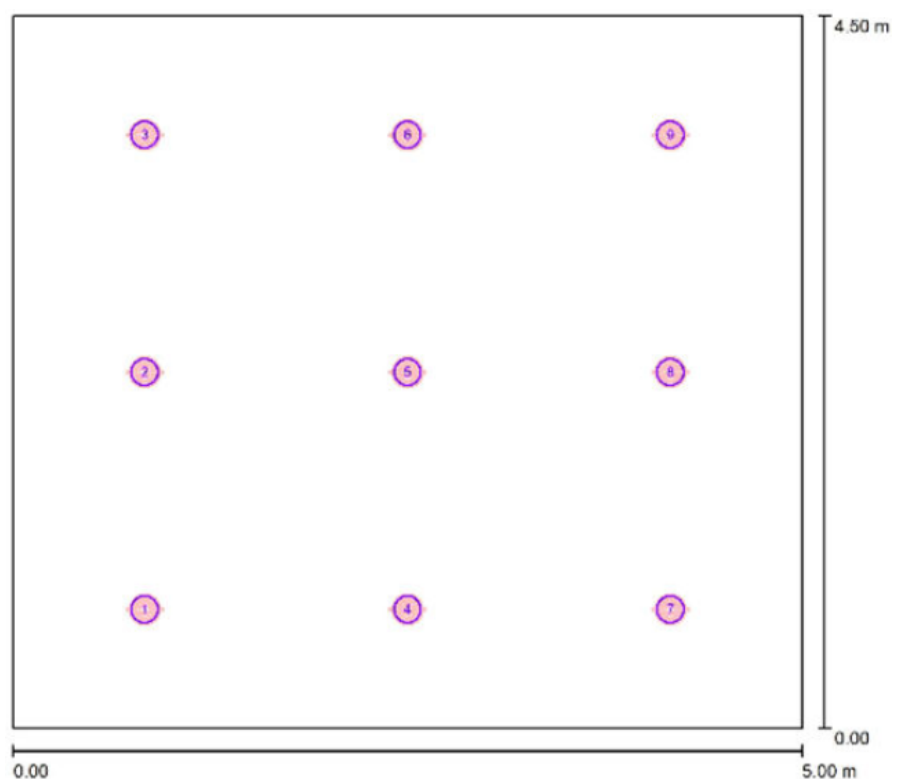

Figure 4. Visual form of the office and arrangement of the luminaires insides the room.

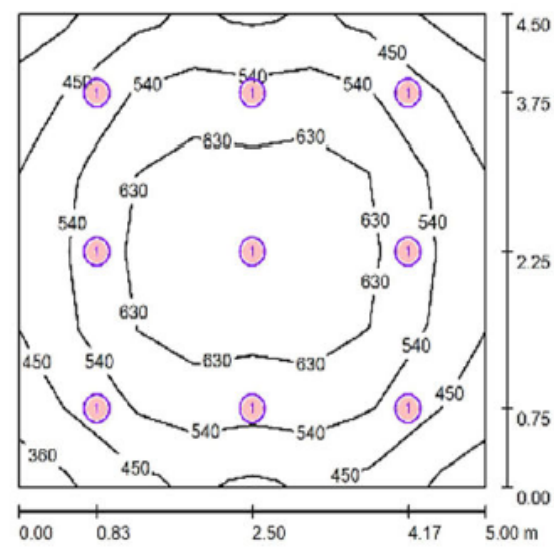

(a)

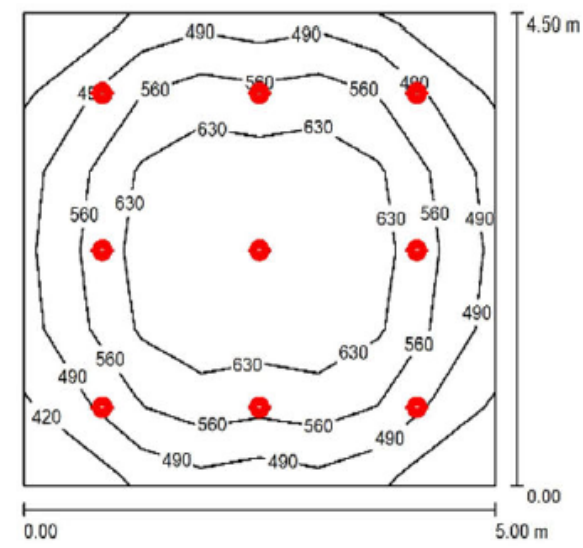

(c)

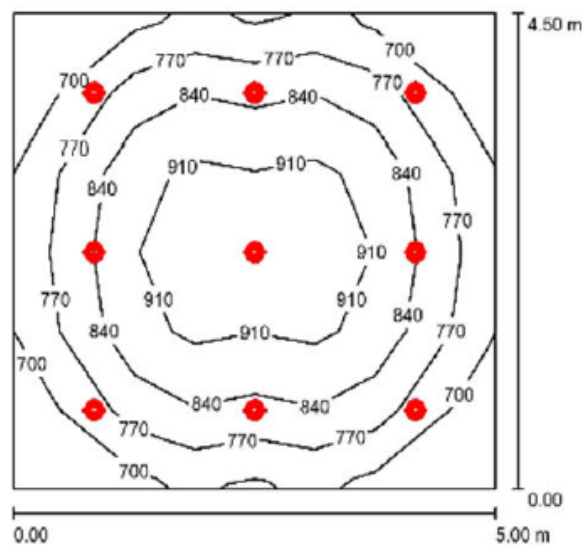

(b)

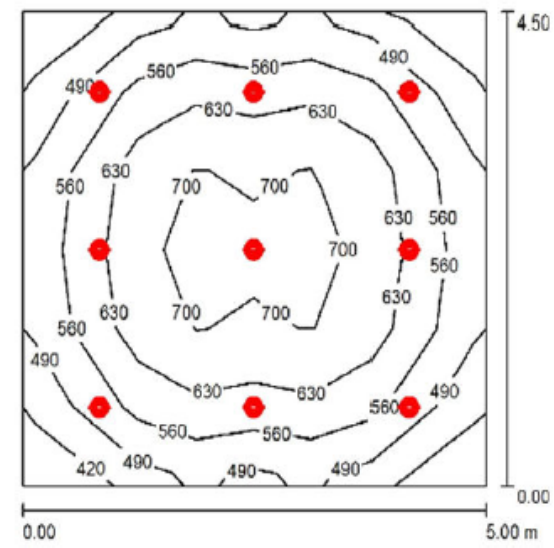

(d)

Figure 5. Illumination isoline for primary colors (a) white (b) red (c) yellow (d) blue. 


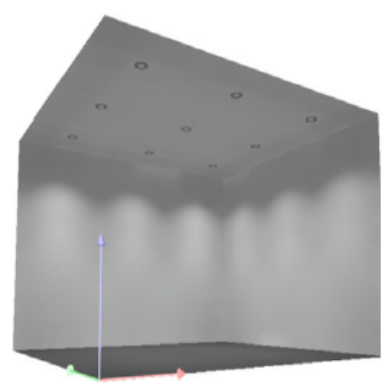

(a)

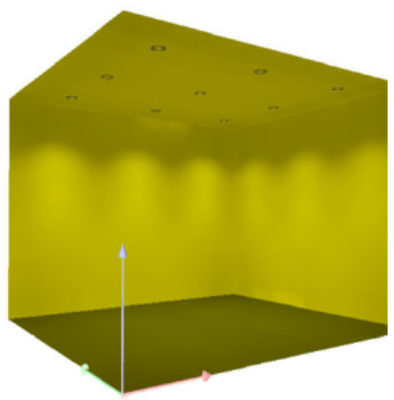

(c)

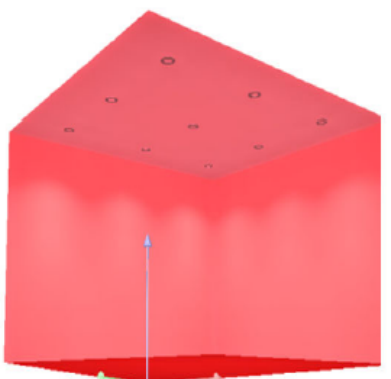

(b)

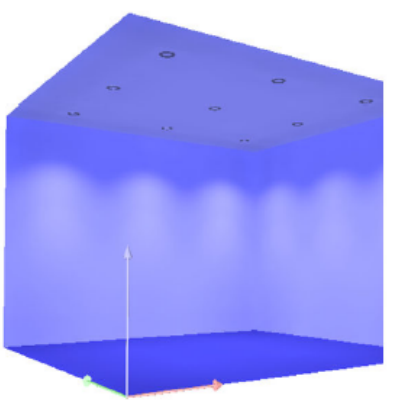

(d)

Figure 6. 3-D rendering for primary color (a) white/black (b) red (c) yellow (d) blue.

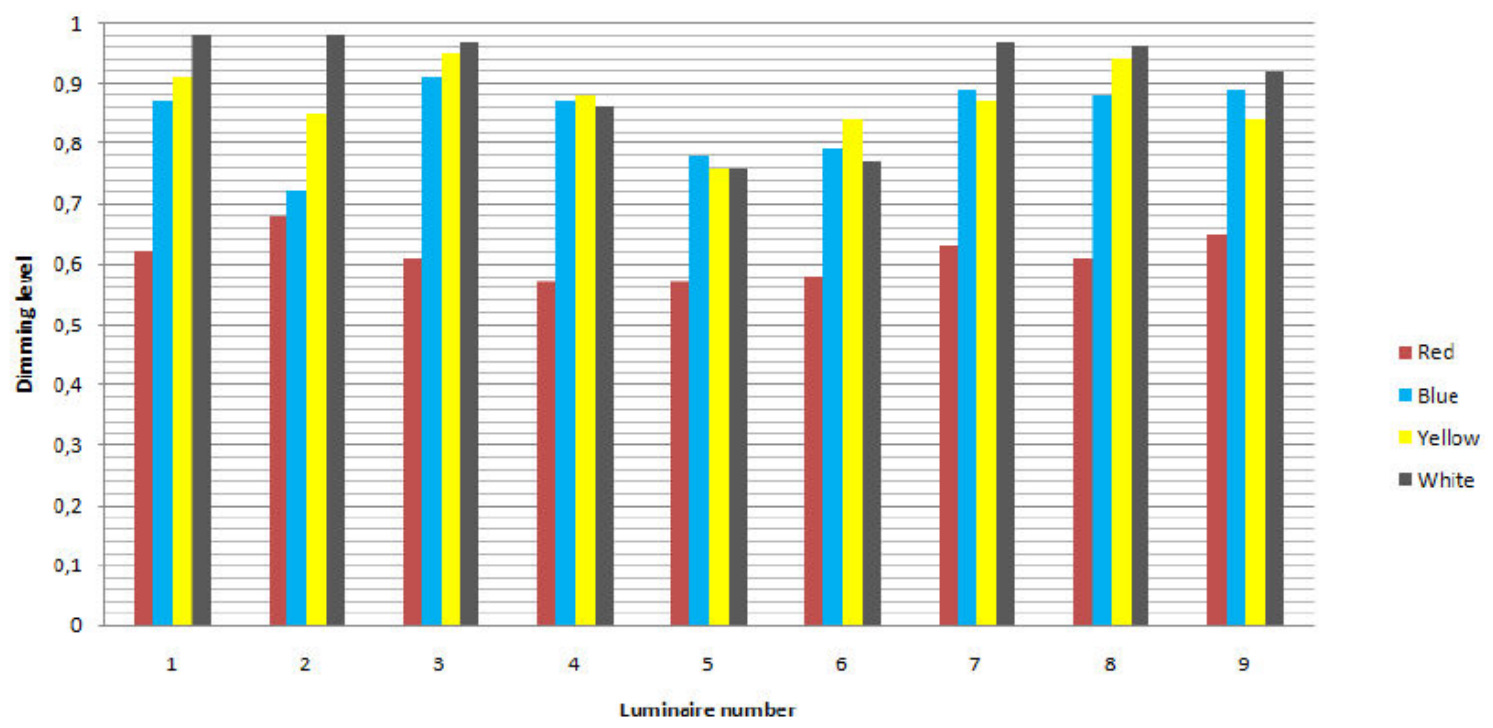

Figure 7. Graph variation of dimming level $\alpha$ according to color choice.

Subject to $\frac{\sum_{i=1}^{48} \sum_{j=1}^{9} \alpha_{j} \rho_{k} \omega_{i j}^{\prime}}{48} \geq 500$ and $0 \leq \alpha_{k} \leq 1$.

A variant of NSGA III [42] was developed in Matlab 2014a [43] to generate a Pareto front that contains optimal solutions. These solutions optimize all of the objectives functions subject to constraint. When running the NSGA III algorithm, it is observed that, to accelerated convergence, the lower value of coefficient $\alpha_{k}$ can be chosen beyond 0.3 because it is difficult to have higher uniformity with a coefficient lower than this critical value. The value of the parameters used are shown in Table 3. The stopping criteria are fixed by the number of generations.

\section{Results and Discussions}

The simulation of the lighting levels is carried out in DIALux. To have fairly uniform values, the colors of the different office walls (floor, walls, and ceiling) are chosen 
identical and can be seen in Figure 6 which gives the 3D rendering after simulation. The isoline curves are shown in Figure 5 for the different colors and one can notice the varying levels of illumination at each square meter of the room. To have a wider range of variation, the secondary colors have been associated with the primary ones. These results show that the neutral colors (white, black and gray) give the lowest levels of illumination while the primary red color gives the highest level. The other colors (blue, yellow, green, purple and orange) allow to appreciate the order of variation of these levels of illumination in the range bounded by the red color at the top and the neutral colors at the bottom of the scale. It should be noted that all the colors that result from a mixture of a primary color with the red one(let say purple or orange for example), have a high level of illumination but less than that obtained with the red color. To understand the high value of the green color compared to the yellow which is here the primary color that gave the lowest level of illumination, we can go back to the literature. Indeed, the green color is often classified as a primary color and the yellow as a secondary color arising from the mixture between green and blue[29]. However, this does not have a major influence on our study since it is part of the intermediate levels knowing that the boundaries are already defined. It is therefore found that the choice of colors has a significant influence on the level of illumination of a room.

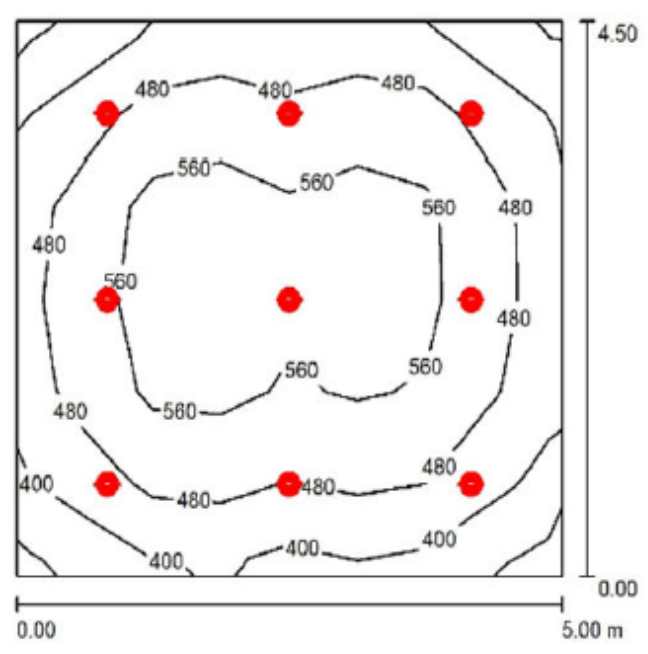

(a)

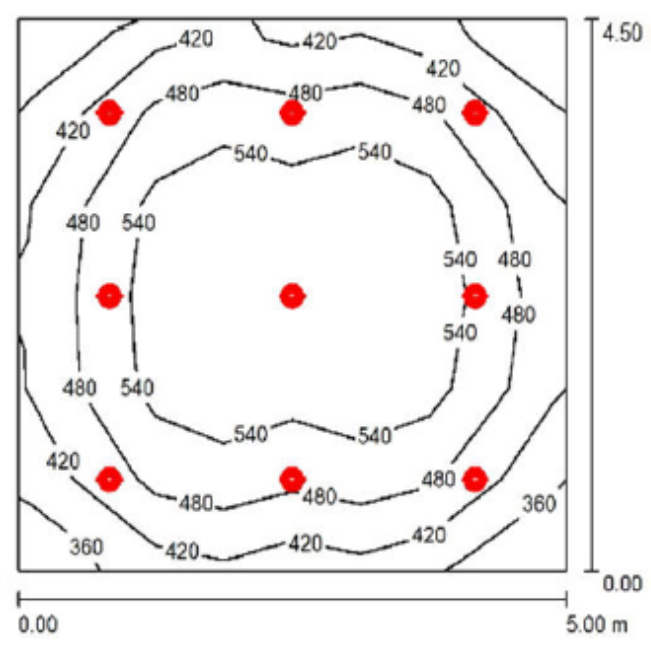

(c)

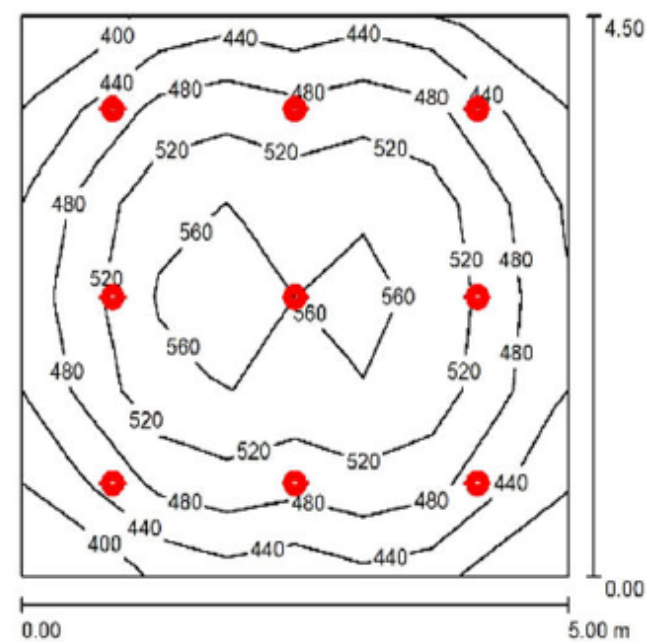

(b)

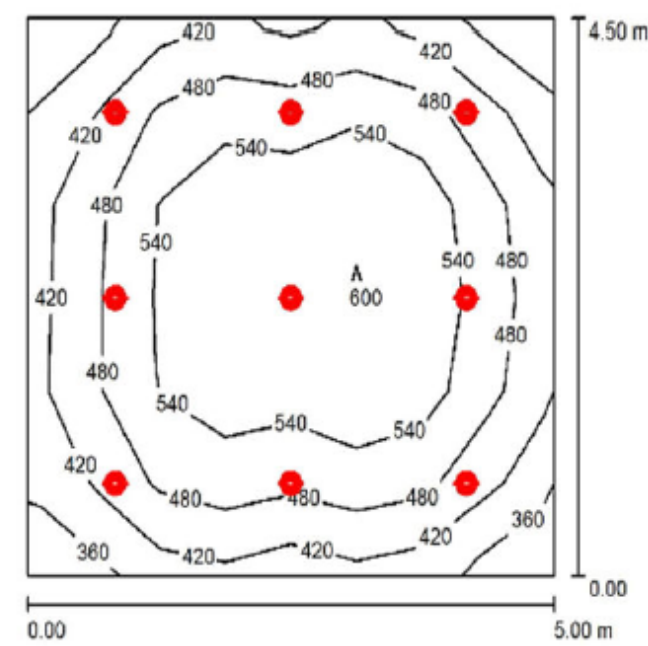

(d)

Figure 8. Isolines with different primary color contour plot of the illuminance accross the room (optimal solutions) (a) white/black (b) red (c) yellow (d) blue. 
The EN 12464 standard sets the minimum permitted lighting levels for visual comfort in the different types of rooms. In our case(room office), it is fixed at 5001x for average illumination and 0.6 for uniformity. With neutral colors, we are closer to these normative values. However, savings can be achieved by reducing the extra lux to rest to a value equal to 5001x. These extra lux represent the surplus of energy. In our experiment, the red color has the highest extra lux(3181x). We then use the genetic algorithm NSGA III implemented under MATLAB and parameterized as shown in Table 3, to obtain the optimal coefficients $a_{k}$. These coefficients are then used in DIALux to have the corresponding $E_{m}$ and $g_{0}$ values. When these values are in accordance with the specifications that have been set, these coefficients are validated as the optimal gradation levels sought. Several computations yield the values depicted in Table 4. Figure 7 confirms visually that the lowest dimming levels for the nine luminaires are obtained for the red color while the highest levels are obtained for the white color.

Table 3. Parameter of NSGA III.

\begin{tabular}{ll}
\hline Parameter & Parameter value \\
\hline Number of individual & 40 \\
Number of generations & 200 \\
Crossover probability & 0.5 \\
Mutation probability & 0.5 \\
Mutation Rate & 0.02 \\
Number of Decision Variables & 9 \\
\hline
\end{tabular}

Table 4. Parameter value after NSGA III optimization and corresponding light simulation.

\begin{tabular}{|c|c|c|c|c|c|c|c|c|c|c|c|c|c|}
\hline \multirow{2}{*}{ Color } & \multicolumn{9}{|c|}{ Coefficient $\alpha_{k}$} & \multirow{2}{*}{$E_{m}$} & \multirow{2}{*}{$g_{0}$} & \multirow{2}{*}{$\mathbf{J 1}(\alpha)$} & \multirow{2}{*}{$\mathbf{J} 2(\alpha)$} \\
\hline & $\alpha_{1}$ & $a_{2}$ & $\alpha_{3}$ & $\alpha_{4}$ & $\alpha_{5}$ & $\alpha_{6}$ & $\alpha_{7}$ & $\alpha_{8}$ & $\alpha_{9}$ & & & & \\
\hline White & 0.98 & 0.98 & 0.97 & 0.86 & 0.76 & 0.77 & 0.97 & 0.96 & 0.92 & 500 & 0.553 & 8.2 & 0.155 \\
\hline Red & 0.62 & 0.68 & 0.61 & 0.57 & 0.57 & 0.58 & 0.63 & 0.61 & 0.65 & 500 & 0.786 & 5.5 & 0.110 \\
\hline Blue & 0.87 & 0.72 & 0.91 & 0.87 & 0.78 & 0.79 & 0.89 & 0.88 & 0.89 & 500 & 0.712 & 7.6 & 0.268 \\
\hline Yellow & 0.91 & 0.85 & 0.95 & 0.88 & 0.76 & 0.84 & 0.87 & 0.94 & 0.84 & 500 & 0.699 & 7.9 & 0.158 \\
\hline
\end{tabular}

Table 5. Optimal ligthing power consumption under different color.

\begin{tabular}{|c|c|c|}
\hline Color & Global Optimal dimming coefficient $(J 1(\alpha))$ & Percentage of energy saved \\
\hline White or Black & 8.2 & $9 \%$ \\
\hline Red & 5.5 & $39 \%$ \\
\hline Blue & 7.6 & $16 \%$ \\
\hline Yellow & 7.9 & $12 \%$ \\
\hline
\end{tabular}

The optimization algorithm, did not only bring back the illumination levels to exactly 500lx but also, improved uniformity as shown in Figure 8 (compared to Figure 5) and Table 4 (compared to Table 2).

The ultimate objective being the reduction of energy consumption, Table 5 shows for the primary and neutral colors used, the range of variation of this economy in power consumed which ranges from $9 \%$ to $39 \%$. It can, therefore, be concluded that depending on the color used, the energy savings can be very high. It should be noted that it would be equally possible to reduce the number of luminaires by keeping the gradation levels close to one, but a change of color after completion of the project would entail additional costs related to the increase of the luminaires. The choice of gradation is, therefore, more appropriate for buildings that could undergo modifications in the future.

\section{Conclusion}

Starting from the problem of reducing the consumption of lighting in buildings, we proposed within this work the influence of colors(floors, walls, and ceiling) with optimization process based on genetic algorithms, in particular, the NSGA III. The problem is modeled as a multi-objective optimization problem, with the main variables being the dimming level of the luminaires and the choice of the colors of the different parts of the room. Knowing that the main factors influencing the illumination of a room are the level of illumination and its uniformity, the objective functions defined seek the minimization of the level of illumination and the maximization of the uniformity by locating their values in the zone prescribed based on the EN 12464 standard. These objectives should lead to a reduction in the energy consumption of buildings. A case study, highlighting the application of the optimization model developed in an office illuminated by 9 LED luminaires recessed in the ceiling of the room, has shown that it is possible to reach according to the chosen 
color, energies ranging from $9 \%$ to $39 \%$ with gradation factor increasingly reduced. The choice of the primary red color is the one that allowed to have the greatest optimization. This model is of great importance for building lighting designers as it will allow them to save energy from the design phase of lighting projects by keeping, at best by improving, the visual comfort of the users. The association with other optimization methods of this model will have the advantage of making it more robust and reduce the human intervention in the process of choosing the optimal solution.

\section{Acknowledgements}

The first author would like to thank Eng. René Blaise Tekendo and Dr. Theophile Fozin for provoking this investigation, some correction and for many informative discussions.

\section{References}

[1] K. Beddiar and J. Lemale. Bâtiment Intelligent Et Efficacité Energetique Optimisation, nouvelles technologies et BIM. Dunod, 2016.

[2] Consumption of energy, 2017. https://ec.europa.eu/eurostat/statisticsexplained/index.php ?title=Archive $:$ Consumption of energy.

[3] Jelena Popović-Gerber, Jesus Angel Oliver, Nicolás Cordero, Thomas Harder, José A Cobos, Michael Hayes, Seán Cian OMathuna, and Erich Prem. Power electronics enabling efficient energy usage : Energy savings potential and technological challenges. IEEE transactions on power electronics, 27(5) :2338-2353, 2012.

[4] V.-Kitio. Intégration des mesures defficacité énergétique et de conservation des ressources dans les normes de construction au cameroun, Juillet 2014.

http://arpedac.org/wpcontent/ uploads/2017/04/ProjetONUHabitat-MiHDU-vincent-Kitio.pdf.

[5] Marie-Claude Dubois and ?ke Blomsterberg. Energy saving potential and strategies for electric lighting in future north european, low energy office buildings : A literature review. Energy and buildings, 43(10) :25722582, 2011.

[6] Pierre-René Bauquis. A reappraisal of energy supply and demand in 2050. Oil and Gas Science and Technology, 56(4) :389-402, 2001.

[7] Jae-Wook Lee, Hyung-Jo Jung, Ji-Young Park, JB Lee, and Yoonjin Yoon. Optimization of building window system in asian regions by analyzing solar heat gain and daylighting elements. Renewable energy, 50 :522-531, 2013.
[8] G Baldinelli, F Asdrubali, C Baldassarri, F Bianchi, F DAlessandro, S Schiavoni, and C Basilicata. Energy and environmental performance optimization of a wooden window : A holistic approach. Energy and buildings, 79 :114-131, 2014.

[9] Youssef Bichiou and Moncef Krarti. Optimization of envelope and hvac systems selection for residential buildings. Energy and Buildings, 43 (12) :3373-3382, 2011.

[10] Gianluca Rapone and Onorio Saro. Optimisation of curtain wall facades for office buildings by means of pso algorithm. Energy and Buildings, 45 :189-196, 2012.

[11] Ramzi Ouarghi and Moncef Krarti. Building shape optimization using neural network and genetic algorithm approach. Ashrae transactions, 112 (1), 2006.

[12] Daniel Tuhus-Dubrow and Moncef Krarti. Geneticalgorithm based approach to optimize building envelope design for residential buildings. Building and environment, 45(7) :1574- 1581, 2010. 10 (2), 181C192.

[13] Eddy Prianto and Patrick Depecker. Optimization of architectural design elements in tropical humid region with thermal comfort approach. Energy and buildings, 35 (3) :273-280, 2003.

[14] Francesco Asdrubali, Francesco DAlessandro, and Samuele Schiavoni. A review of unconventional sustainable building insulation materials. Sustainable Materials and Technologies, 4 :1-17, 2015.

[15] Jiangjiang Wang, Zhiqiang John Zhai, Youyin Jing, and Chunfa Zhang. Particle swarm optimization for redundant building cooling heating and power system. Applied Energy, 87 (12) :3668-3679, 2010.

[16] Ebrahim Solgi, Zahra Hamedani, Ruwan Fernando, Henry Skates, and Nnamdi Ezekiel Orji. A literature review of night ventilation strategies in buildings. Energy and Buildings, 2018.

[17] Jens Pfafferott, Sebastian Herkel, and Matthias Wambsgan?. Design, monitoring and evaluation of a low energy office building with passive cooling by night ventilation. Energy and buildings, 36 (5) :455-465, 2004.

[18] Jared Landsman. Performance, prediction and optimization of night ventilation across different climates. 2016.

[19] Natasa Djuric, Vojislav Novakovic, Johnny Holst, and Zoran Mitrovic. Optimization of energy consumption in buildings with hydronic heating systems considering thermal comfort by use of computer-based tools. Energy and Buildings, 39 (4) :471-477, 2007. 
[20] Amar M Khudhair and Mohammed M Farid. A review on energy conservation in building applications with thermal storage by latent heat using phase change materials. Energy conversion and management, 45 (2) :263-275, 2004.

[21] Juan F De Paz, Javier Bajo, Sara Rodrłguez, Gabriel Villarrubia, and Juan M Corchado. Intelligent system for lighting control in smart cities. Information Sciences, 372 :241-255, 2016.

[22] Nandha Kumar Kandasamy, Giridharan Karunagaran, Costas Spanos, King Jet Tseng, and Boon-Hee Soong. Smart lighting system using ann-imc for personalized lighting control and daylight harvesting. Building and Environment, 139 :170C180, 2018.

[23] Wa Si, Harutoshi Ogai, Katsumi Hirai, Hidehiro Takahashi, and Masatoshi Ogawa. An improved pso method for energy saving system of office lighting. In SICE Annual Conference 2011, pages 1533-1536. IEEE, 2011.

[24] M Corcione and L Fontana. Optimal design of outdoor lighting systems by genetic algorithms. Lighting Research \& Technology, 35 (3) :261-277, 2003.

[25] Francisco Chueco, Fernando Lpez, and Miguel Bobadilla. Technical and economic evaluation of fluorescent and led luminaires in underground mining. a case study : New mine level of el teniente. Energy and Buildings, 93 :16$22,2015$.

[26] ZizhenWang and Yen Kheng Tan. Illumination control of led systems based on neural network model and energy optimization algorithm. Energy and Buildings, 62 :514521, 2013.

[27] Evangelos-Nikolaos D Madias, Panagiotis A Kontaxis, and Frangiskos V Topalis. Application of multi-objective genetic algorithms to interior lighting optimization. Energy and Buildings, 125 :66-74, 2016.

[28] Souffo Tagueu Merim and Ndzana Beno?t. Lighting optimisation control of fluo/led systems using neural network and mathematical model. International Journal of Electrical Engineering \& Technology, 10 (4) :47-59, 2019.

[29] Lawrence D Woolf. Confusing color concepts clarified. The Physics Teacher, 37(4) :204-206, 1999.

[30] Light and lighting-lighting of work places ? part 1: Indoor work places, June 2011. European Committee for Standardization.
[31] Yao-Jung Wen and AM Agogino. Control of wirelessnetworked lighting in open-plan offices. Lighting Research \& Technology, 43 (2) :235-248, 2011.

[32] Michael Fischer, Kui Wu, and Pan Agathoklis. Intelligent illumination model-based lighting control. In 2012 32nd International Conference on Distributed Computing Systems Workshops, pages 245-249. IEEE, 2012.

[33] Shigang Cui, Huimin Lv, Xingli Wu, Yongli Zhang, and Lin He. Optimization of plant light source based on simulated annealing particle swarm optimization algorithm. In 2018 Chinese Control And Decision Conference (CCDC), pages 700-703. IEEE, 2018.

[34] Ralph Evins. A review of computational optimisation methods applied to sustainable building design. Renewable and sustainable energy reviews, 22 :230-245, 2013.

[35] Shady Attia, Mohamed Hamdy, William OBrien, and Salvatore Carlucci. Assessing gaps and needs for integrating building performance optimization tools in net zero energy buildings design. Energy and Buildings, 60 :110-124, 2013.

[36] Kalyanmoy Deb, Amrit Pratap, Sameer Agarwal, and TAMT Meyarivan. A fast and elitist multiobjective genetic algorithm: Nsga-ii. IEEE transactions on evolutionary computation, 6(2) :182-197, 2002.

[37] Hisao Ishibuchi, Ryo Imada, Yu Setoguchi, and Yusuke Nojima. Performance comparison of nsga-ii and nsga-iii on various many-objective test problems. In 2016 IEEE Congress on Evolutionary Computation (CEC), pages 3045-3052. IEEE, 2016.

[38] Haitham Seada and Kalyanmoy Deb. U-nsga-iii : A unified evolutionary algorithm for single, multiple, and many-objective optimization. COIN report, 2014022, 2014.

[39] Dali - digital addressable lighting interface. http://www.dali-ag.org.

[40] Dialux documentation. https://www.dial.de/en/dialux/.

[41] Kalyanmoy Deb and Himanshu Jain. An evolutionary many-objective optimization algorithm using referencepoint-based nondominated sorting approach, part i: solving problems with box constraints. IEEE Transactions on Evolutionary Computation, 18(4) :577601, 2014.

[42] Matlab 2014 documentation. https://fr.mathworks.com/products/global-optimization.html. 